\title{
Research on the Method of Optimal PMU Placement
}

\author{
http://dx.doi.org/10.3991/ijoe.v9iS7.3189 \\ XU Jian-jun, XU Yan-chao, YAN Li-mei*, ZHAO Hai-long, SUN Zhi-gang, BAI Li-li, XIE ming-xia \\ Northeast Petroleum University, Daqing, P.R. China
}

\begin{abstract}
Taking the full network observability of power system and the least number of PMU as objective, to appearing fault situation in the grid, this paper proposes Differential Evolution and Particle Swarm Optimization (DEPSO) algorithm in view of the system failure rate. The improved DEPSO algorithm is global optimization, the algorithm takes the constraint condition of fault rate into account during the course of seeking optimal solutions. At the end, through the examples show that the algorithm compares with the existing optimization methods, which can reduce the number of $P M U$ configuration and achieve completely observability of the system, at the same time, and stable operation of the system, through the simulation results verify feasibility and valibity of the algorithm.
\end{abstract}

Index Terms-PMU; observability; DE algorithm; PSO algorithm; optimal placement

\section{INTRODUCTION}

Synchronized Phasor Measurement Unit (Phasor Measurement Unit, PMU) is a new type of phasor measurement unit based on the Global Positioning System (Global Positioning System, GPS). In order to realize the synchronous data collection of various nodes of the power system, it uses high-precision timing signals for GPS system. PMU device has high measurement accuracy, it can directly measure the node voltage phasors and branch current phasors as other measurements, especially the phase angle can also be measured [1]. If the PMU devices can be configured in all the nodes of power system, then the entire power system will be fully observable, and the grid flow will no longer need any iterative calculation. However, as the PMU technology is not mature enough and still has some economic issues, it is impossible for the power system to configure each node with PMU device. As a result, the optimal of PMU has been extensive researched by scholars all over the word. Cho K S was the first one who considered this issue and proposed using Simulated Annealing (SA) algorithm [2]. But because of its observability judge adopted the conventional numerical methods which requires a lot of matrix calculations with slowly speed and easily affected results by numerical computation accuracy. In addition, the convergence speed of SA algorithm is too slow which affects the practicality of bulk power system. On the basis of depth-first-search method, Jia Hongjie proposed an improved depth-firstsearch (DFS) algorithm through improved optimization rules [3]. This method was used to get the solution of optimal PMU allocation problem which considered the influence of generators, loads and network structure as well as other factors. It also uses the relationship between its network structure and observability which considers the factors of degree of nodes importance, PMU direct measurement, PMU virtual measurement and the impact of PMU distribution. The improved algorithm can realize the observability of power system with less PMU number of installations which can effectively improve the solving efficiency and accuracy of the algorithm. However, the given method only conducted from observability without considering the system redundancy requirements which is the short of the algorithm. By analyzing the node power equations proposes the scheme of PMU optimal placement in order to directly solve the power flow [4-6]. But this method only considers how to get the unknown voltage of adjacent nodes and unknown status of associated node which injected power is zero for the known nodes of power equations. Without considering the PMU may be redundantly configured caused by the situation that some voltage phasors can be directly solved. Nowadays, as the implement of the "Smart Grid" strategy, the safe and stable operation of the grid is also facing new challenges. In order to contain large area blackout accident and evolution, this paper proposes to consider the case of system failure rate and use a hybrid optimization algorithm of Differential Evolution and Particle Swarm Optimization (DEPSO). By making use of the global search capability of DE algorithm and PSO algorithm to achieve the goals to reduce the PMU numbers and keep the complete observability of power system, at the same time improve the convergence speed of the algorithm and ensure the safety and reliability operation of system.

\section{THE OBSERVABILITY OF POWER SYSTEM}

\section{A. The observability of system}

Power system observability is defined as the systematic measurement sets with the amount, types and distribution which is sufficient to solve the current state of the system. In the network topology of the whole power system, the node which voltage phasors can be measured directly or indirectly obtained are called observability nodes, otherwise called a non-observation nodes. If all nodes in the system are observability nodes, the system is completely observed, if not the system is incompletely observed. The system observability can be divided into algebra observability and topology observability [7].

(1) Algebra observability 
Algebra observability mainly judged by checking whether the triangular decomposition of information matrix will get zero main elements. For a power system which has $N$ nodes and $m$ measured values, its algebra observability can be described as:

$$
z=H x+v
$$

Where $z$ is $m$ dimension measurement phasor, $H$ is $m \times(2 N-1)$ dimensional measurement Jacobian matrix, $x$ is $(2 N-1)$ dimension voltage status phasor, $v$ is $m$ dimension noise phasor.

If the measurement Jacobian matrix $H$ is full rank and good state, which can meet $\operatorname{rank}(H)=2 N-1$, then the whole power system called algebra observability. However, it has a large analysis computation of algebra observability and the accuracy will be easily affected by cumulative errors.

(2) Topology observability

Topology observability is to check whether the measurement configuration set can establish a full rank spanning tree to cover all the nodes in the whole network. In the perspective of graph theory, the whole power system can be regarded as a graph $G=(V, E)$ which has $N$ nodes and $b$ edges, where $V$ is set of graph nodes, $E$ is set of graph edges, which respectively correspond to the system bus set and branch set. Measuring network constitutes a measure subgraph $G^{\prime}=\left(V^{\prime}, E^{\prime}\right)$, where $V^{\prime} \subseteq V, E^{\prime} \subseteq E$. If graph $G^{\prime}$ and $G^{\prime}$ are fit for $V \subseteq V^{\prime}$, which is graph $G^{\prime}$ contains all the nodes of graph $G^{\prime}$, then the whole system called topology observability [8].

\section{B. The judgment conditions for system observability}

Since the power system topology exists a high degree of sparsity, configuring PMU on part nodes can realize the observability of the system. The judgment conditions for system observability are:

(1) If the voltage and current phasors of one end of the branch are known, then the other end voltage phasor can be calculated through the branch constraint equation;

(2) If the voltages of both ends of a branch are all known, then the branch current can be calculated indirectly;

(3) If no configuration PMU measurement of zero injection node and its relates branch current only one unknown, then the branch current can be obtained according to the KCL equation;

(4) The all adjacent nodes voltage phasor of unknown zero injection node are known, the zero injection node voltage phasor can be calculated by nodal equations.

So PMU measurement sets can be defined as directly measurements which are each PMU node voltages and associated branch currents; other node voltages and currents can be calculated indirectly through the known measurements by Ohm's law and Kirchhoff's law. According to the four conditions given above we can get the following conclusions:

(1) If any adjacent node numbers are 2 then two adjacent injection node of zero injection node can be observed, which is the node can be observed;

(2) If any node was configured PMU, then the node can be observed, each adjacent nodes of this node can also be observed;
(3) If any zero injection node of $N$ adjacent nodes can be observed, and the $\mathrm{N}-1$ adjacent nodes can be observed, then its other adjacent nodes can also be observed;

(4) If all the adjacent node voltage phasors of the unknown zero injection node are known, then the node voltage phasor can be calculated by nodal equations, so the node is called observed nodes.

\section{PMU OPTIMIZATION PROBLEM}

\section{A. PMU optimal allocation conditions}

Configuring PMU devices need to meet the conditions in the power system as follows:

(1) Complete observability of power grid;

(2) Configuring minimum number of PMUs;

(3) Considering grid failure rate and configuring PMU device on the high failure rate node firstly to ensure safe and stable operation of the grid.

\section{B. Outlet number and node incidence matrix}

Outlet number refers to the nodes number connected to the node. As an example of WSCC9-node system:

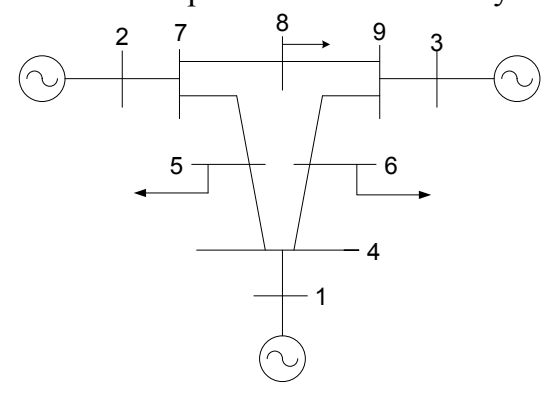

Figure 1. WSCC9-node system

According WSCC9-node system diagram known outlet number matrix:

$$
\operatorname{out}[9]=\left\{\begin{array}{lllllllll}
1 & 1 & 1 & 3 & 2 & 2 & 3 & 2 & 3
\end{array}\right\}
$$

According to the nodes connection in the system, the node system converted to a binary matrix, which is $N \times N$ dimension correlation matrix.

Establishing the node incidence matrix as follows:

$$
A_{9}=\left[\begin{array}{lllllllll}
1 & 0 & 0 & 1 & 0 & 0 & 0 & 0 & 0 \\
0 & 1 & 0 & 0 & 0 & 0 & 1 & 0 & 0 \\
0 & 0 & 1 & 0 & 0 & 0 & 0 & 0 & 1 \\
1 & 0 & 0 & 1 & 1 & 1 & 0 & 0 & 0 \\
0 & 0 & 0 & 1 & 1 & 0 & 1 & 0 & 0 \\
0 & 0 & 0 & 1 & 0 & 1 & 0 & 0 & 1 \\
0 & 1 & 0 & 0 & 1 & 0 & 1 & 1 & 0 \\
0 & 0 & 0 & 0 & 0 & 0 & 1 & 1 & 1 \\
0 & 0 & 1 & 0 & 0 & 1 & 0 & 1 & 1
\end{array}\right]
$$

By the matrix: $a_{i j}=1$ means nodes 1 and $\mathrm{j}$ are connected; $a_{i j}=0$ means nodes $\mathrm{i}$ and $\mathrm{j}$ are not connected. By outlet number matrix and node correlation matrix shows: 9th node outlet number is 3 , if 9 th node is installed a PMU device, 3, 6, 8 nodes are observable.

\section{The selection of the initial value}

Based on fully observable of the power system and to ensure safe and reliable operation of the grid, this article 
selects high failure rate node installing PMU device preferentially to real-time monitoring power grid operation status. Therefore, the initial PMU configuration rules as follows:

(1) Zero injection node does not configure PMU device if adjacent node number is one and adjacent nodes are two;

(2) Selecting the node with the highest failure rate priority configuration PMU, as the current best position;

(3) Configure the necessary PMU configuration, if the system is still not fully observed, select the node associated with largest tributary as a new configuration node in the remaining unobserved nodes.

\section{PMU optimal placement mathematical model}

By observable determine conditions to analyze the power system, selecting the node with the highest failure rate of the network is installed PMU device firstly, new adjacent observable nodes can be obtained through the observable principle of the node. In this paper, to ensure maximum observability of the power system as the premise for a $\mathrm{N}$-node system, PMU optimal allocation problem is to determine the minimum PMU number and the set of optimal installation locations by fully considering the grid failure rate factors, the following expressions can be described:

$$
\begin{aligned}
& \min y=\sum_{i=1}^{N} x_{i} \\
& \sum_{i \in P} f\left(x_{i}\right) \geq \sum_{i=1}^{N} x_{i} \\
& \text { s.t. } U_{i}=1 \quad i=1,2, \mathrm{~L}, N
\end{aligned}
$$

Where $y$ is PMU number, $N$ is system bus number, $x_{i}=1$ means node $i$ is configured PMU device, $x_{i}=0$ means node $i$ is not configured PMU device. The dimension of the solution to the optimization problem is equal to bus number of the power system and is expressed by binary form, i.e. the value of each dimension can only be 0 or 1 . When a node is installed PMU, the corresponding position of the solution is to take 1. Equation (2) is minimizing the number of PMUs, unequal constraint (3) is to meet complete observability.

From the perspective of optimization, the system observability problem depends on two variables, one for the configuration PMU number and another for the collection of configuration PMU position $P(n)$. PMU optimal allocation is nonconsecutive nonlinear optimization problems, usually there are a large number of local extreme points need to be solved with iterative optimization algorithm [9]. Intelligent optimization algorithm with a global search capability can solve this complex nonlinear optimization problem, this paper uses the Differential Evolution (DE) and Particle Swarm Optimization (PSO) hybrid algorithm.

\section{PMU OPTIMIZATION ALGORITHM}

This thesis combines DE algorithm with PSO algorithm. After considering system failure rate, PMU measurements and virtual measurement factors, an improved algorithm is proposed.
According to the configuration principle of PMU to analyze the system, select the nodes as preferred installation of PMU, then work out new adjacent observable node through the principle of observable node. The configuration optimization of PMU is a class of problem which is discrete high-dimensional nonlinear combinatorial optimization problem. Generally there will be many feasible solutions. The thesis evaluates feasible solution considering power grid failure factors.

\section{A. Differential Evolution algorithm}

DE algorithm possesses mutation, crossover and selection operation. DE algorithm generates variant individual based on difference vector among random selection parent individuals. Then crossover operation is performed between parent individuals and the newly generated variation individuals to generate test individuals. Selecting operation is to choose the size of the fitness values between parent individuals and test individuals, and then get new individuals.

(1)Mutation operation

Mutation is a key step of DE algorithm to generate individuals. Mutation operation randomly selects an individual from population as base vector, and make other two different individuals as difference vector. Using the difference variation and reorganization operator of evolutionary strategy, there are $M$ generated new individuals. The formula of mutation operation is following:

$$
\begin{aligned}
& v_{i j}(t+1)=x_{b e s t j}(t)+F_{i}(t) \cdot\left(x_{i 1 j}(t)-x_{i 2 j}(t)\right) \\
& w_{i j}(t+1)=\left(x_{i 3 j}(t)+x_{i 4 j}(t)\right) / 2
\end{aligned}
$$

Where adaptive mutation factor is $F_{i}(t)=\beta\left(e^{\lambda}-1\right)$, $\lambda=\frac{T_{\max }}{T_{\max }+t}, \lambda$ is controlling element, $T_{\max }$ is the biggest evolution algebra, $t$ is current evolution algebra, $\beta$ is a constant, $\beta \in[0.2,0.6], i, i 1, i 2, i 3, i 4$ are not equal and $i 1, i 2, i 3, i 4$ are any random positive integer in $[1$ , $\mathrm{M} / 2$ ], $x_{\text {best }}$ is the current optimal position.

(2)Crossover operation

Crossover operation can increase the diversity of population, specific operation as follows:

$$
\begin{aligned}
& u_{i j}(t+1)= \begin{cases}v_{i j}(t+1) & \text { if }(\operatorname{rand}[0,1] \leq C R) \\
x_{i j}(t) & \text { else }\end{cases} \\
& C R=C R_{\min }+\frac{\left(C R_{\max }-C R_{\min }\right) t^{2}}{T_{\text {max }}^{2}}
\end{aligned}
$$

Where $u_{i j}(t+1)$ is test individual, $C R$ is crossover probability operator, $C R_{\max } 、 C R_{\min }$ are the largest and smallest crossover probability factor, in this thesis $C R_{\min }=0.1, C R_{\max }=1.0$.

Crossover probability factor can balance the global and local search ability very well.

(3) Select operation

In the differential evolution algorithm, selecting operation takes the greedy strategy - choose one-on-one between test individuals and the original parent individuals. Determinant of choosing is comparing their fitness values, operation equation as follows: 


$$
x_{i j}(t+1)=\left\{\begin{array}{lc}
u_{i j}(t+1) & f\left(f\left(u_{i j}(t+1)\right) \leq f\left(x_{i j}(t)\right)\right) \\
x_{i j}(t) & \text { else }
\end{array}\right.
$$

Where $x_{i j}(t+1)$ is the position of the $i$ th among the new generation of individuals. $f$ is fitness function.

\section{B. Particle Swarm Optimization}

PSO algorithm assumes that the potential solution of the optimization problem as a no mass and no volume bird, called particle. PSO search mechanism: the search process of particle tracking two "extreme" to update their information, one is individual extreme that is the optimal solution found by particle itself; another is the global extreme that the optimal solution found by the entire current population currently. Particle follow these two optimal solutions, in accordance with certain rules update their velocity and position [10]. PSO formulas are:

$$
\begin{gathered}
v_{i j}(t+1)=w(t) v_{i j}(t)+c_{1} r\left(p_{i j}(t)-x_{i j}(t)\right) \\
+c_{2} r\left(p_{g j}(t)-x_{i j}(t)\right) \\
x_{i j}(t+1)=x_{i j}(t)+v_{i j}(t+1) \\
w(t)=w_{\min }+\left(w_{\max }-w_{\min }\right) \frac{T_{\max }-t}{T_{\max }}
\end{gathered}
$$

Where $p_{i j}$ is individual extreme, $p_{g j}$ is global extreme, $w$ is inertia weight factor, $w_{\max } 、 w_{\min }$ are maximum and minimum inertia weight, $T_{\max }$ is maximum evolution generation, $c_{1}, c_{2}$ are accelerating factors, generally $c_{1}=c_{2}, r \in(0,1)$ random number.

\section{Improved DEPSO algorithm}

Differential and particle swarm algorithms are heuristic algorithm, the local search accuracy difference algorithm is superior to PSO. PSO convergence speed is fast, establishment parameter is less, easy to implement and so on. Optimization PSO algorithm has good performance, but because of the late evolutionary algorithms due to the reduced particle diversity easy to fall into local optimum. Differential evolution algorithm through mutation, crossover and selection operations generate new populations to achieve the optimal solution through iterative search, DE has a powerful function of the maintenance of the population diversity and search capabilities, but the convergence speed is slow in latter part. This article will combine DE algorithm and PSO algorithm, and introduced in the algorithm determines evolutionary factor $H$, as the ratio of previous generation iterative optimal solution and the optimal solution of the current iteration ratio, that is:

$$
H=\frac{f\left(x_{i j}(t-1)\right)}{f\left(x_{i j}(t)\right)}
$$

Where $H \geq 1$, the greater $H$ is, the faster the speed is. With the increasing of iteration numbers, $H$ is held on one, but did not find an optimal solution, the algorithm stagnantes or falls into local optimum.

Flow chart of DEPSO hybrid algorithm is shown in Figure 2.

The basic steps of DEPSO hybrid algorithm in this paper are as follows:

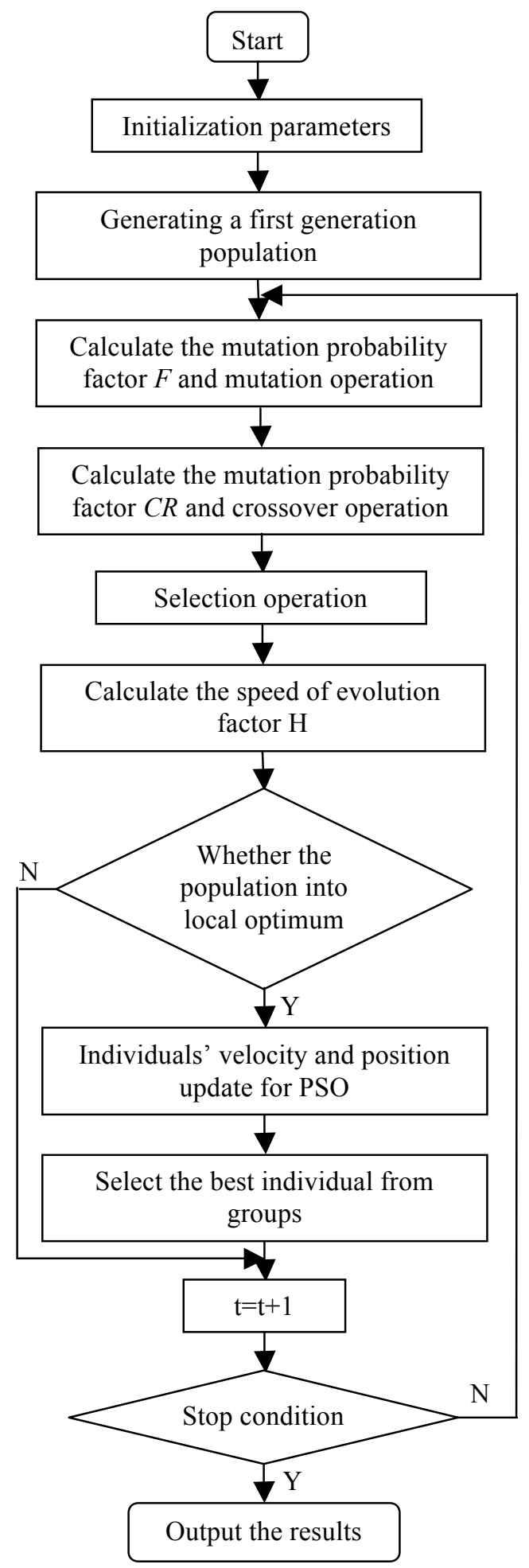

Figure 2. Fig.2 Flow chart of DEPSO hybrid algorithm

(1) Initialization parameters. Set the population size $N$, maximum evolution algebra $T_{\max }$, and the related parameters of DE algorithm and PSO algorithm, including maximum and minimum inertia weight $w_{\max }, w_{\min }$, acceleration factors $c_{1}, c_{2}$, largest and smallest crossover probability $C R_{\max }, C R_{\min }$, control factor $\lambda$, evolution speed factor $H$, mutation probability factor $F$, make counter $t=0$; 
(2) Generating a first generation population. Set the position and speed of individual populations, $x_{\text {best }}$ is optimal particle position of initial population;

(3) Calculate the current value of the mutation probability factor $F$ for DE algorithm mutation operation;

(4) Calculate the current value of the crossover probability factor $C R$ for $\mathrm{DE}$ algorithm crossover operation;

(5) Individuals on the population objective function value calculated for $\mathrm{DE}$ algorithm selection operation;

(6) Calculate the speed of evolution factor $\mathrm{H}$, determine whether the population into local optimum. If it is converted into local optimum step (7), if it is not fall into local optimum is transferred to step (9);

(7) PSO to optimize the operation carried out on the population so that individuals' velocity and position update;

(8) Select the best individual from groups;

(9) $t=t+1$;

(10) Detecting whether satisfy the stop condition. If satisfied then stop, output the results, otherwise, converted to the step (3).

\section{EXAMPLE AND ANALYSIS}

This paper proposes DEPSO algorithm considering the node failure rate of the power system, and are applied to IEEE14-node system and New England 39-node system to be emulated to verify the effectiveness and feasibility of the algorithm.

\section{A. IEEE14-node system}

IEEE14-node system is shown in Figure 3.



Figure 3. IEEE14-node system

7th node is zero power injection node not need to configure PMU device, and 6th node with the highest failure rate need to configure PMU device firstly. Simulation shows that system is considerable through installing PMU devices in the node 2, 6, 9. The simulation results can be seen from Table 1, the proposed algorithm with other algorithms described in the literature comparing, DEPSO algorithm has obvious advantages, both to reduce the computation time and ensure optimal results.

Tab.1 Comparison of optimal placement scheme of PMU for IEEE14-node system.
TABLE I.

COMPARISON OF OPTIMAL PLACEMENT SCHEME OF PMU FOR IEEE14NODE SYSTEM

\begin{tabular}{lccc}
\hline Method & Time/s & $\begin{array}{c}\text { Number of } \\
\text { required } \\
\text { PMUs }\end{array}$ & $\begin{array}{c}\text { Locations of } \\
\text { required } \\
\text { PMUs }\end{array}$ \\
\hline DEPSO & 0.480 & 3 & 269 \\
PSO & 0.699 & 3 & 256 \\
DFS & 0.701 & 6 & 146814 \\
SA & 2.423 & 4 & 4569 \\
MST & 4.226 & 3 & 269 \\
\hline
\end{tabular}

Figure 4 shows that this paper proposes hybrid DEPSO algorithm has improved significantly than the classical PSO algorithm in the convergence rate.

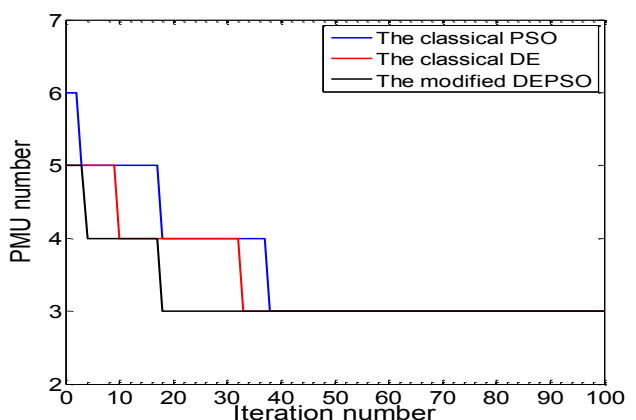

Figure 4. Comparison of the classical PSO and DE, the modified DEPSO for IEEE14-node system

\section{B. New England 39-node system}

New England 39-node system is shown in Figure 5, configuration shows in Table 2 and Figure 6.

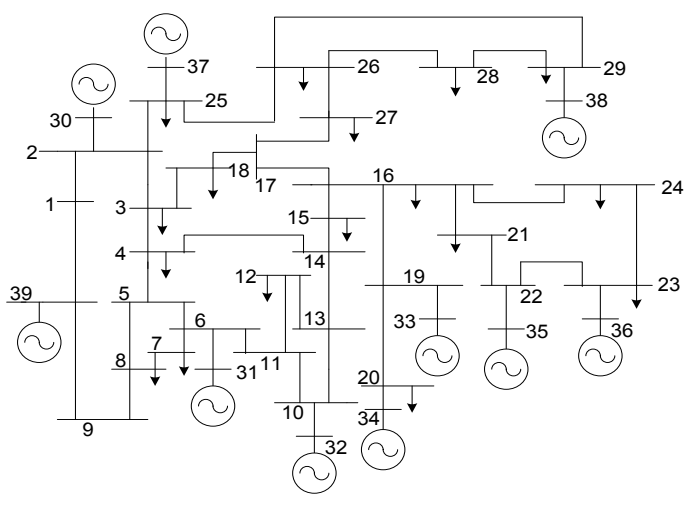

Figure 5. New England39-node system

TABLE II.

COMPARISON OF OPTIMAL PLACEMENT SCHEME OF PMU FOR NEW ENGLAND39-NODE SYSTEM

\begin{tabular}{|c|c|c|c|}
\hline Method & Time/s & $\begin{array}{l}\text { Number of } \\
\text { required } \\
\text { PMUs }\end{array}$ & $\begin{array}{c}\text { Locations of } \\
\text { required PMUs }\end{array}$ \\
\hline DEPSO & 0.466 & 8 & $\begin{array}{c}3812162023 \\
2529\end{array}$ \\
\hline PSO & 0.702 & 8 & $\begin{array}{c}3810162023 \\
2529\end{array}$ \\
\hline DFS & 0.661 & 16 &  \\
\hline
\end{tabular}




\begin{tabular}{cccc}
\hline \multirow{2}{*}{ SA } & \multirow{2}{*}{11.679} & 9 & 238121620 \\
& & 232529 \\
MST & 49.992 & 9 & 238101620 \\
& & 232529 \\
\hline
\end{tabular}

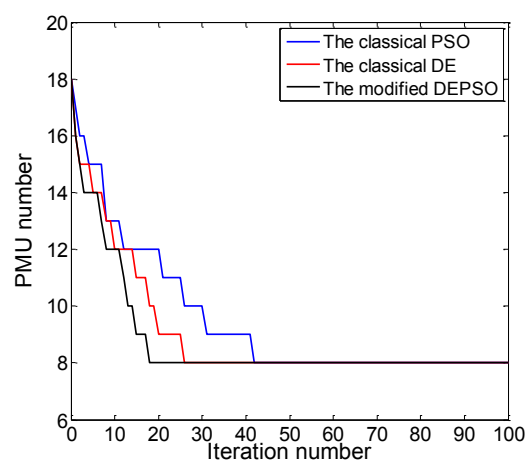

Figure 6. Comparison of the classical PSO and DE, the modified DEPSO for New England39-node system

\section{CONCLUSION}

This paper proposes an improved DEPSO algorithm for solving PMU optimal allocation problem, it considers the case of system failure as its advantage and the probability of node configuration PMU is changed by the failure rate of system. Calculating the evolutionary factor is to get rid of the possibility of the algorithm falling into local optimal solution, and its core idea is to take advantage of the node failure, between system topology and its observability relationship, PMU direct measurement, virtual measurement and other factors, to set a larger installation probability in the higher failure rate and a larger qualification degree. The algorithm is not only ensure fully observable but also minimum number of PMUs, it provides the failure rate as a comparison parameter and effectively improves the efficiency and accuracy of the solution algorithm, the examples prove this algorithm is feasible, while ensuring the system is safe and stable operation.

\section{ACKNOWLEDGMENT}

1. This work was supported by Natural Science Foundation of Heilongjiang Province (E201260).

\section{REFERENCES}

[1] Cho K S, Shin J R, Hyun S H. Optimal Placement of Phasor Measurement Units with GPS Receiver. In: Fifth International Conference on Power System Control. Seoul (South Korean):2001.

[2] Yang Yi. Research on the PMU Optimal Allocation in Power System. Wuhan: Hubei University of Technology, 2012.
[3] Jia Hongjie, Lv Yinghui, Zeng Yuan, et al. Optimal PMU placement in power system. JOURNAL OF ELECTRIC POWER SCIENCE AND TECHNOLOGY, 2010, 25(1): 54-59.

[4] Wang Jia,Yao Jiangang, Sun Qian,et al. Hybrid algorithm of optimal PMU placement in power systems. Computer Engineering and Applications, 2013, 49(3):267-270.

[5] Wang Jialin, Xia Li,Wu Zhengguo. Optimal PMU Allocation of Shipboard Hybrid AC/DC System. Power System Technology, 2012, 36(10): 81-86.

[6] Guo Peiying, Hao Hongyan, Deng Ying,et al. Optimal Configuration of PMU in Power System Considering the biggest measurement redundancy[J]. Journal of Northeast Dianli University, 2010, 30(2): 31-35.

[7] Wang Jialin, Xia Li, Wu Zhengguo,et al. New Method of Optimal PMU Placement of the Power Systems. Proceedings of the Chinese Society of Universities for Electric Power System and its Automation, 2011, 23(6): 1-5.

[8] Yang Chunhua, Qian Xiaoshan, Gui Weihua. Hybrid algorithm of chaotic differential evolution and particle swarm optimization. Application Research of Computers, 2011, 28(2): 439-441.

[9] Qiao Xiaoyan, Qiao Kun, Meng Xin, et al. Optimal PMU Configuration in Power System. Power System and Clean Energy, 2010, 26(10): 26-30.

[10] Liu Bin, Huang Qun, Li Bo, et al. Application of Improved Binary Particle Swarm Optimization Algorithm in PMU Placement. Proceedings of the CSU-EPSA, 2010, 22(2): 5-9.

\section{AUTHORS}

XU Jian-jun is with the Department of Electrical Information Engineering, Northeast Petroleum University, Daqing, P.R. China (e-mail: junjianxu@163.com).

XU Yan-chao is with the Department of Electrical Information Engineering, Northeast Petroleum University, Daqing, P.R. China China (e-mail: 123939273@qq.com).

YAN Li-mei is with the Department of Electrical Information Engineering, Northeast Petroleum University, Daqing, P.R. China, and is the Correspondence Author.(e-mail: yanlimeidaqing@163.com).

ZHAO Hai-long is with the Department of Electrical Information Engineering, Northeast Petroleum University, Daqing, P.R. China. (e-mail: 6504628@163.com)

SUN Zhi-gang is with the Department of Electrical Information Engineering, Northeast Petroleum University, Daqing P.R. China. (e-mail: 13845902468@163.com)

BAI Li-li is with the Department of Electrical Information Engineering, Northeast Petroleum University, Daqing, P.R. China. (e-mail: bailili211@163.com)

XIE ming-xia is with the Department of Electrical Information Engineering, Northeast Petroleum University, Daqing, P.R. China. (e-mail: xiemingxia@163.com)

Submitted, 17 January, 2013. Published as resubmitted by the authors on 16 May, 2013. 